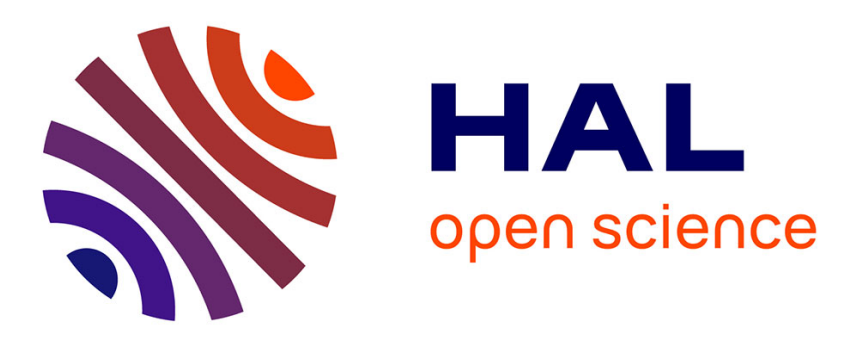

\title{
Tomographic diffractive microscopy and multiview profilometry with flexible aberration correction
}

Hui Liu, Jonathan Bailleul, Bertrand Simon, Matthieu Debailleul, Bruno Colicchio, Olivier Haeberlé

\section{- To cite this version:}

Hui Liu, Jonathan Bailleul, Bertrand Simon, Matthieu Debailleul, Bruno Colicchio, et al.. Tomographic diffractive microscopy and multiview profilometry with flexible aberration correction. Applied optics, 2013, 53 (4), pp.748-755. 10.1364/AO.53.000748 • hal-00998064

\section{HAL Id: hal-00998064 \\ https://hal.science/hal-00998064}

Submitted on 30 May 2014

HAL is a multi-disciplinary open access archive for the deposit and dissemination of scientific research documents, whether they are published or not. The documents may come from teaching and research institutions in France or abroad, or from public or private research centers.
L'archive ouverte pluridisciplinaire HAL, est destinée au dépôt et à la diffusion de documents scientifiques de niveau recherche, publiés ou non, émanant des établissements d'enseignement et de recherche français ou étrangers, des laboratoires publics ou privés. 


\title{
Tomographic diffractive microscopy and multiview profilometry with flexible aberration correction
}

\author{
H. Liu, J. Bailleul, B. Simon, M. Debailleul, B. Colicchio, and O. Haeberlé* \\ Laboratoire Modélisation Intelligence Processus et Systèmes MIPS-EA2332, Université de Haute-Alsace, \\ 61 rue Albert Camus, 68093 Mulhouse cedex, France \\ ${ }^{*}$ Corresponding author: olivier.haeberle@uha.fr
}

Received 15 November 2013; revised 20 December 2013; accepted 20 December 2013; posted 23 December 2013 (Doc. ID 200550); published 30 January 2014

\begin{abstract}
We have developed a tomographic diffractive microscope in reflection, which permits observation of sample surfaces with an improved lateral resolution, compared to a conventional holographic microscope. From the same set of data, high-precision measurements can be performed on the shape of the reflective surface by reconstructing the phase of the diffracted field. Doing so allows for several advantages compared to classical holographic interferometric measurements: improvement in lateral resolution, easier phase unwrapping, reduction of the coherent noise, combined with the high-longitudinal precision provided by interferometric phase measurements. We demonstrate these capabilities by imaging various test samples. (C) 2014 Optical Society of America

OCIS codes: (090.0090) Holography; (090.1000) Aberration compensation; (100.6640) Superresolution; (110.6955) Tomographic imaging; (070.0070) Fourier optics and signal processing.

http://dx.doi.org/10.1364/AO.53.000748
\end{abstract}

\section{Introduction}

In classical microscopy using incoherent illumination and detection (wide-field, phase-contrast or differential interference contrast), the recorded image results from a complex interaction of the incoherent illuminating light with the specimen. As a consequence, the contrast, which is observed, permits efficient morphological studies, but does not directly deliver quantitative information on the opto-geometrical characteristics of the specimen. In particular, the specimen's optical index of refraction distribution may be difficult to reconstruct. On the contrary, recording the diffracted field in both amplitude and phase allows for the reconstruction of the specimen permittivity map or index of refraction distribution, using an adapted model of diffraction. Thanks to its coherent imaging capacities, digital holographic microscopy provides useful information about a large range of transparent, as well as

$1559-128 \mathrm{X} / 14 / 040748-08 \$ 15.00 / 0$

(C) 2014 Optical Society of America reflective, specimens [1]. One drawback is the resolution limitation associated with coherent imaging [2]. Different systems have been developed to improve the resolution in coherent imaging. The accessible spectrum can be enlarged by moving the sensor, combining different holograms at different camera positions to obtain a large digital hologram, thus improving the effective numerical aperture (NA) [3]. Synthetic aperture systems, based on gratings used to downshift the object frequencies, have also been reported in one dimension [4] and two dimensions [5]. Single exposure systems üsing spatial multiplexing have also been proposed. They use a source array and an incoherent addition of multiple holograms [6].

Another possibility is to downshift the object frequencies into the detection range of the system by using illumination wavefronts tilted with respect to the object. In this case, the process usually relies on the Fourier shift theorem; the object function is modulated, which causes a spectrum shift, bringing back spatial frequencies in the bandpass of the system. For example, a tilt can be introduced in the object [ $\underline{7-9}]$ or in the plane wave illumination [10 $\underline{12}$. 
In the case of plane-wave illumination, the object function is multiplied by a phase ramp, whose slope is given by the director cosine of the illumination. In Fourier space, the spectrum is then convolved with a Dirac delta function localized at the carrier frequency, inducing a spectrum shift. In this situation, the principle of resolution improvement is similar to tomographic diffractive imaging (TDM) [13-16], but in TDM, the synthetic aperture process is associated with a backpropagation, or a 3D Fourier spectrum synthesis, leading to direct 3D imaging, instead of two-dimensional (2D) imaging. Other tomographic techniques can be used, such as rotation of the specimen $[17,18]$; however, the resolution is improved only along the optical axis and limited by the precision of the multiple-image registration, as maintaining a high-precision rotation, compatible with interferometric measurement, is difficult to realize. The TDM setup may, of course, be used for observing quasi-2D objects, i.e., object weakly structured in the depth direction. The system can perform both phase or amplitude imaging on $2 \mathrm{D}$ or $3 \mathrm{D}$ objects. In the $2 \mathrm{D}$ case, the process of backpropagation is not useful and leads to useless additional calculations. Fast synthetic aperture imaging can be realized if the process is restricted to $2 \mathrm{D}$ calculations. In addition, on thin objects, the classical TDM imaging has no real interest because the resolution in the $z$ direction is not sufficient to image such small details. On the contrary, for purely reflective objects, the elevation can be directly obtained from the phase information, as in standard profilometry.

\section{Principle of 2D Spatial Spectrum Enlargement and Phase Imaging}

\section{A. Phase Imaging}

In topography, the classical formula used to calculate the height profile $h$ under normal illumination is $[19]$

$$
h(x, y)=\frac{\lambda}{4 \pi}\left(\phi(x, y)+\phi_{r}(k)\right),
$$

where $h(x, y)$ is the height to be determined, $\phi(x, y)$ the phase measured by interferometry, $\lambda$ the wavelength of illumination in the medium of refractive index $n$ (usually 1 in air), and $\phi_{r}(k)$ is the phase change at the reflection.

\section{B. Synthetic Aperture}

The proposed process of digital 2D synthetic imaging on weakly diffracting objects is described in Fig. 1 . The complex field $E_{i}$ (image plane) is extracted from the holograms $H_{\theta i}$ and then Fourier transformed to obtain $S_{i}$, a shifted version of the spectrum in $\Gamma$, the original frequency support. The specular component $S_{i 0}$ is extracted in this spectrum, which allows for phase normalization by the complex division $S_{i}(f x, f y) / S_{i 0}$. The frequencies are then shifted, with respect to the coordinates of the specular beam (zero

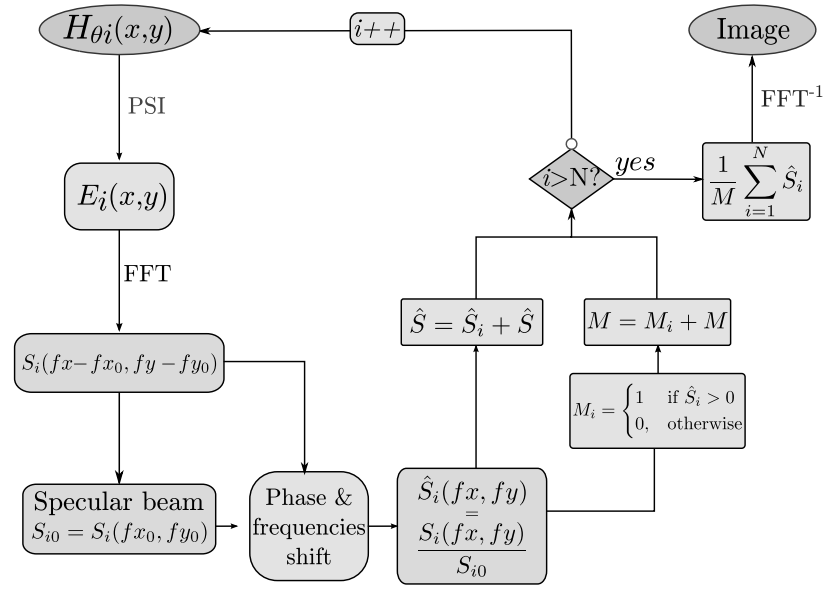

Fig. 1. 2D synthesis process for amplitude imaging. $H$, hologram; $E$, complex optical field; $S$, spectrum in original support $\Gamma$. $\hat{S}$, normalized spectrum in synthesized support $\Gamma^{\prime}$. M, redundancy map used to average the merged data; $i$, index of the illumination angle.

frequency). Thus, one obtains a centered and normalized spectrum $\hat{S}_{i}$, in an enlarged frequencies support $\Gamma^{\prime}$. Normalized spectra are then summed iteratively on $\mathrm{N}$ holograms in this enlarged support. Because of overlaps between each spectrum, one determines a map of data occurrence $\mathrm{M}$, which gives the occurrence weight of each spatial frequency, to be used for averaging the data. The final image is then obtained by an inverse Fourier transform.

The final bandpass can theoretically be doubled, compared to a normal incidence illumination only, leading to better imaging of small details $[\underline{3}-\underline{6}, \underline{8}, 9]$. In addition, on smooth objects, increasing the $\mathrm{NA}$ of the profilometric system permits to collect higher-angle reflected beams, which translates into higher-slope measurement possibilities [19].

Figure 2 illustrates the whole process in the Fourier space, when imaging an embossed compact disc (CD) surface. Figure 2(a) shows the spectrum obtained for one oblique illumination, while Fig. 2(b) shows the composite spectrum obtained for eight evenly spaced illuminations at the highest tilting angle. Note the doubling of the spectrum bandpass. Figure 2(c) shows the composite spectrum for 100 illuminations. While the spectrum is still marginally filled compared to Fig. 2(b), note the smoothing effect resulting from the averaging procedure.

\section{Experimental Setup}

Figure 3 depicts our multimode system, which can be used in reflexion [20], but also (for the sake of clarity, not shown on the figure) transmission [13], and fluorescence [21]. Throughout this work, we only consider the reflection setup. An interferometer has been added on the rear port of an Olympus IX71 inverted microscope: a coherent source (B\&W Tek, $20 \mathrm{~mW} \max$, BWB-475 $\lambda_{0}=475 \mathrm{~nm}$ ) is split into an illumination and a reference beam. The length of both arms is adjusted using optical fibers. The phase of the reference beam is controlled by a fast 

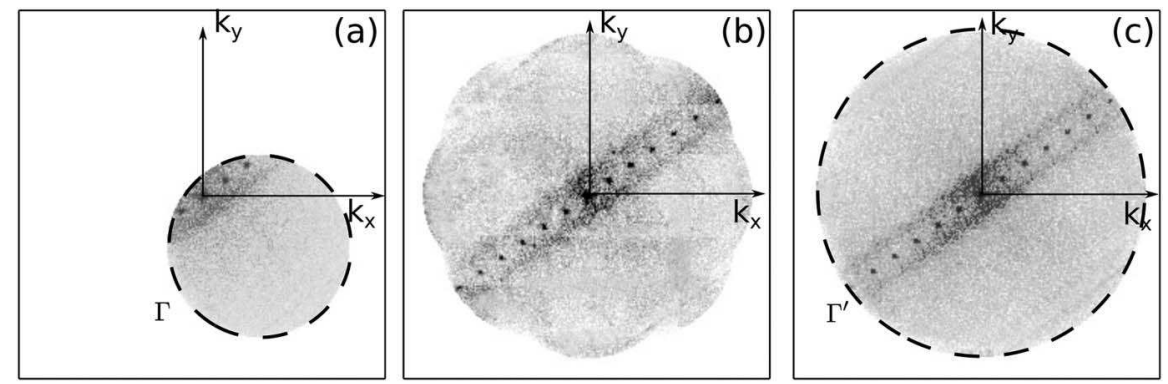

Fig. 2. Spectrum modulus of a $C D$ reconstructed with (a) one illumination ( $\Gamma$ support). (b) Eight illuminations with bandpass enlargement and (c) 100 illuminations with noise averaging ( $\Gamma^{\prime}$ support).

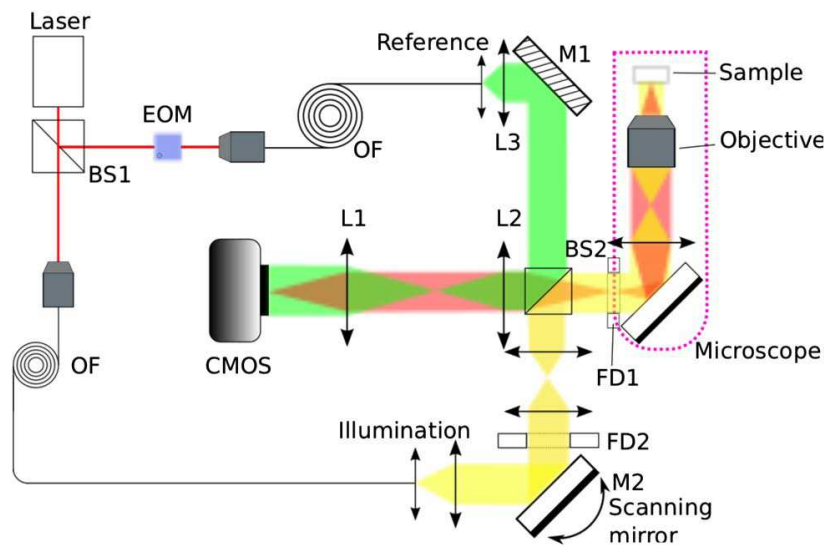

Fig. 3. Experimental setup in reflection mode. BS, beam splitter; EOM, electro-optic modulator; OF, optical fiber; M1, M2, mirror; FD, field diaphragm.

electro-optic modulator (EOM) and its angle by mirror $M_{1}$, so that the holographic setup can be used in phase shifting or off-axis mode. The phase shifting mode (via an EOM) is to be preferred when maximizing the field of view, while the off-axis (via mirror M1) mode allows for optimizing the speed. A fast scanning mirror (Newport, FSM 300) is used to sequentially control the illumination beam. Illumination is performed through a high-NA microscope objective $(\mathrm{NA}=1.4$, oil immersion or $\mathrm{NA}=1.2$, water immersion). The light reflected by the sample is then collected through the microscope objective, and combined with the reference beam via BS2. The telescopic relay formed by lenses L1 and L2 allows for fine adjustment of the sampling on the camera $(1312 \times 1082$ pixel, 68 fps, Photon Focus PHFMV1 CMOS).

The objects under examination in this work are weakly structured in the $z$ direction; the maximum elevation does not exceed one wavelength, and the test pattern used for calibration has a height within the calculated depth of field of the objective microscope, so that the whole object is observed on focus.

In our setup, the additional lateral phase ramp measured on the camera allows for measuring the angle of illumination by identifying the specular beam in Fourier space, which is a Dirac delta function localized at the carrier frequency.
The spatial frequency scanning is performed by controlling the $\left(k_{i x}, k_{i y}\right)$ components of the illumination, maintaining $k_{i z}$ constant

$$
\begin{gathered}
k_{i x}=\left|\vec{k}_{i}\right| \cos \varphi \sin \theta, \\
k_{i y}=\left|\vec{k}_{i}\right| \sin \varphi \sin \theta, \\
k_{i z}=\left(k_{i}^{2}-k_{i x}^{2}-k_{i y}^{2}\right)^{0.5}=\left|\vec{k}_{i}\right| \cos \theta,
\end{gathered}
$$

where $\varphi$ is the azimuthal angle, and $\theta$ is the polar angle, defined by the object plane and the plane of incidence (see Fig. 4). For constant $\theta, k_{i z}$ is kept unchanged and the modulation in the $z$ direction remains the same for all holograms (see Section 4.C). In addition, this scanning allows for minimizing the shadowing effect, which may occur when imaging modulated, purely reflective samples.

On the reference arm, lens $L_{3}$ is used to match the reference wavefront with the illumination wavefront. Even if not perfectly matched, the remaining phase difference between both wavefronts contains only low-frequency aberrations, which can then easily

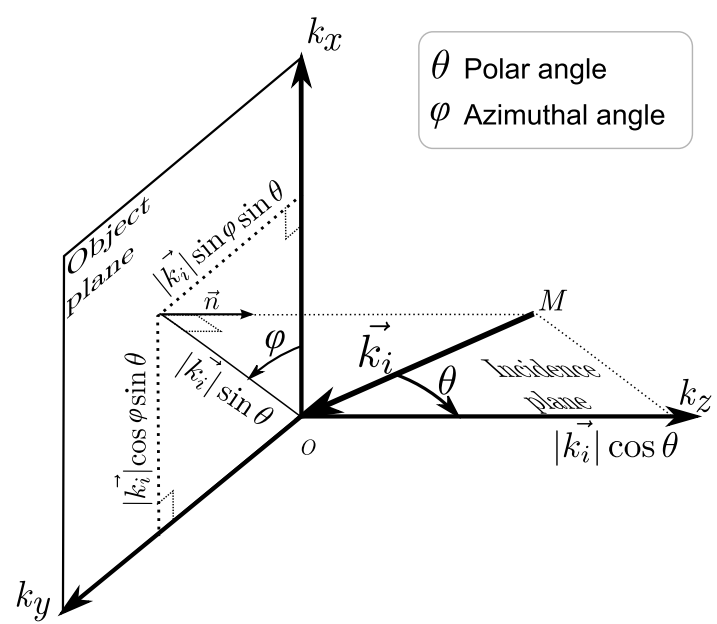

Fig. 4. Notations and coordinate system. In this work, the angular scanning is performed at a fixed polar angle so that $k_{i z}$ remains constant. 
be removed by the numerical procedure described below.

All calculations are performed on a Linux box equipped with an Intel quadcore $i 7$ processor with 16 Gbytes of memory, and programmed in $\mathrm{C}++$ using open source libraries (FFTW, OpenCV).

The real and imaginary parts are extracted from the four phase-shifted holograms, $A, B, C, D$. Twopass averaging ( $\varphi_{1}$ from $A, B, C, \varphi_{2}$ from $B, C, D$ ) is used to reduce the phase noise [19]. The inevitable phase drifts due to angular beam scanning must be compensated to avoid errors when merging the different holograms. In transmission, this can be easily made by using the specular beam [13]. On thin objects, in reflection, common information between successively captured holograms can be used to match the phase: it can be done if the object comprises a flat area, which can be used as a reference plane. The phase shift can then be corrected by matching their common components in the Fourier space, taking benefit of the overlap, which exists between successively captured holograms. The position of each spectrum is corrected and the hologram spectrums are then merged to form the final synthesized spectrum.

\section{Application of Tomographic Diffractive Microscopy and Multiview Profilometry}

In phase imaging, the precision of the measurement can be degraded in different ways, for example by aberrations, speckle noise, or wrong phase unwrapping. When residual aberrations are removed, using multiple tilted wavefronts for topographic imaging has also advantages for improving phase imaging, beside the possibility of synthetic aperture.

\section{A. Automatic Aberration Compensation}

In holographic imaging, the phase difference between the object function and the reference is measured. Any discrepancy or mismatch between both beams, introduced for example by aberrations, will lead to phase measurement errors. This constraint is stronger in phase imaging microscopy than in TDM imaging because the height measurement precision (which can be in the sub-nanometric scale [22]) is much better than the $z$ resolution achieved by TDM (which is in the sub-micrometric range only [20]). In addition, the aberration removal procedure should be at least partially automatic in order to process the multiple holograms to be acquired, contrary to conventional holographic microscopy, using only one illumination.

Aberration compensation usually relies on the optimization of a quality criterium (usually the Strehl ratio combined to an aberration model [23], or the maximization of the specular component in Fourier space [24]), or a reference hologram allowing for the measurement of any departure from a perfect reference wave (i.e., plane or spherical). This reference hologram is obtained by double exposition
[25], or by selecting a profile (for example, a line profile) in a flat area of the sample [26].

We use here a flexible method based on a polynomial approximation of the aberration function by using a random point selection in the background image. Figure 5 depicts the process. First, the object edges are detected using a Canny filter. After a dilatation, a border following algorithm constructs the contours of the object, and a binary mask $m(x, y)$ is created, splitting the space into the object area $(m(x, y)=0)$ and the background area $(m(x, y)=1)$. The original image is multiplied by this binary mask to extract the background. This background is then fitted by a polynomial function using a least square method. The points used for the least square fitting are randomly chosen in the external part of the object, thanks to the binary mask. The final corrected image is obtained by substracting this aberration polynomial to the original image.

In the reflection mode, the process requires a wellknown background surface (a flat surface being the easiest to use); however, with this procedure there is no need for adjustment of the manual parameters of an aberration model. There is also no need for choosing the area used to calculate the aberration polynomial model since it is constructed using random points in the image. Neither double exposition nor manual selection of a profile is required. The minimum number of points to be selected is automatically set to match the type of polynom used in the least square fitting, and, as a consequence, the binary mask does not need to be very precise.

Figure 6 illustrates the procedure, when applied to imaging a commercial $\mathrm{CD}$. The aluminum reflective layer was isolated by dissolving the polycarbonate protective layer with aceton. The first image Fig. 6(a) is an altitude map of the CD bumps obtained from the synthesized phase image and impaired by aberrations. Figure 6(b) shows the automatically extracted binary mask. Figure 6(c) depicts the phase aberration polynom extracted from Fig. 6(a) on the flat reference selected by the mask, here dominated by astigmatism. Figure $6(\mathrm{~d})$ then shows the corrected image. An interesting aspect of this procedure is that any reference background surface may be used, for example, a portion of sphere or paraboloid, if a focusing mirror would need to be observed. The whole

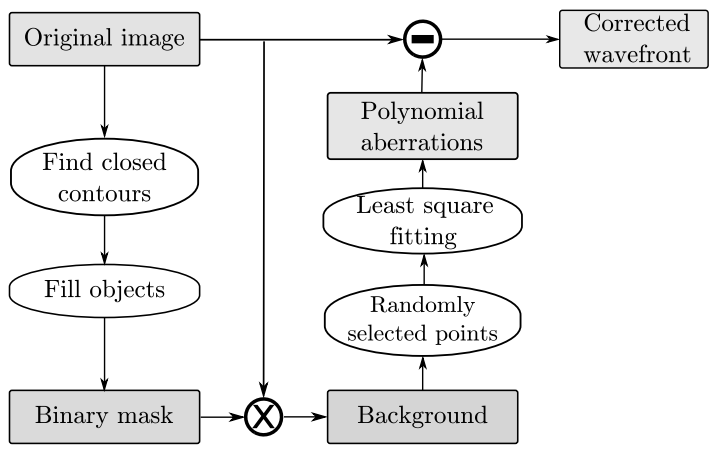

Fig. 5. Process for automatic aberration removal. 


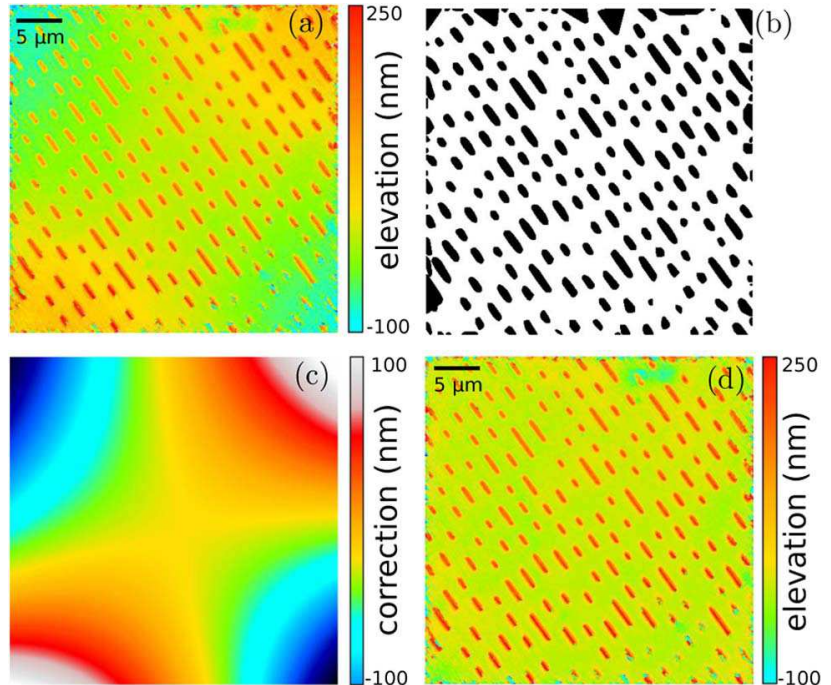

Fig. 6. Imaging of a CD surface. (a) Original image, (b) binary object mask, (c) computed correction map, and (d) final corrected phase image.

process is not demanding from a computational point of view, as the synthetic aperture process takes less than $1 \mathrm{~s}$ for 50 interferograms and the aberration removal less than $0.1 \mathrm{~s}$ per hologram.

\section{B. Noise Reduction}

Temporal averaging can only help for reducing the shot noise [27]. When averaging the common information between successively captured holograms, those holograms are affected by different noise patterns [28] because of the changing illumination. Therefore, as a side-effect of the procedure, it also automatically leads to a reduction of the speckle noise in the final images. The efficiency of the process depends both on the correlation between the different noise patterns and on the number of successive illuminations used for imaging the sample. It can be evaluated by calculating the speckle contrast as a function of the number of holograms.

Figure 7 quantifies the variation of the speckle contrast obtained when combining one to 100 holograms, acquired with circular scanning illumination, and measured when reconstructing images of a VLSI Standards, Inc. STR10-1000P silicon test pattern. This target is platinum coated and consists in $100 \mathrm{~nm}$ high, $5 \times 5 \mu \mathrm{m}$ silicon pitches forming a $10 \times$ $10 \mu \mathrm{m}$ grid on a silicon substrate. The evaluation is performed by analyzing the speckle measured in the images of a flat area of the reference sample [see Fig. 9(a)]. A technique to average speckle noise is to change the speckle pattern by introducing a diffuser in the illumination arm and averaging several holograms, or increasing the acquisition time to integrate the noise pattern. Another approach is to use short coherence length lasers, but this may render the adjustment of the interferometer difficult. Here the noise patterns are automatically modified when the angle of illumination is changed so that no additional element is required to average these

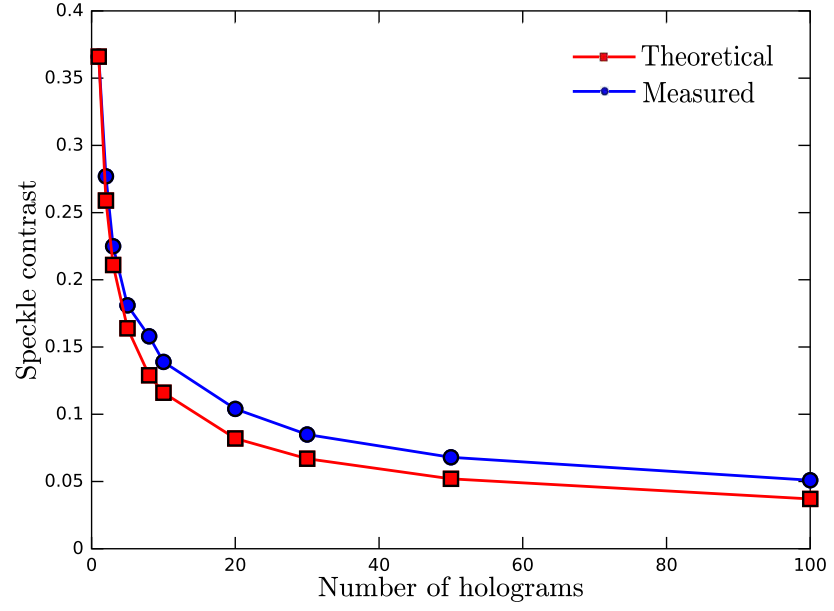

Fig. 7. Evolution of the speckle contrast with the number of illuminations in TDM.

decorrelated noises. A small difference is observed between the theoretical curve for speckle noise reduction [29] and the experimental one, but note that a large diminution of the noise is obtained, which is an interesting side-effect of the multiple illuminations. For fully uncorrelated speckle, theory predicts a noise diminution proportional to the square root of the number of frames [29,30], which is indeed observed. The remaining difference suggests partial correlation between the images acquired with different illumination angles, and/or the presence of a static noise. This technique therefore permits to diminish the noise, using an easy-to-setup interferometer, while allowing for a high sampling of the specimen, contrary to uncoherent interferometers (for example, Shack-Hartmann wavefront sensors), which have an excellent accuracy and dynamic range, but are, up to now, more limited by image pixelation [31].

\section{Phase Unwrapping}

In profilometry, the phase change between two adjacent pixels is limited to a step height equal to $\lambda / 4$ (phase change equal to $\pi$ ). When the change is larger, the phase information is not correctly sampled, which leads to errors in the profile reconstruction. The height limitation $\Delta z_{\min }$ can be severe when using a high-NA objective (immersion objective) to obtain a high lateral resolution. A possibility to overcome this limitation consists simply in tilting the illumination beam. When the illumination beam is tilted, Eq. (1) is modified, and, considering only the modulation along the $z$ direction, the phase difference between the illumination beam and the object beam becomes

$$
h(x, y)=\frac{\lambda}{4 \pi \cos \theta} \phi(x, y),
$$

where $\theta$ is the illumination angle. By controlling the direction of illumination, the quantity $\lambda / \cos \theta$ in Eq. (5) can be varied and then used as an equivalent of an optical frequency scanning [32]. This can help 


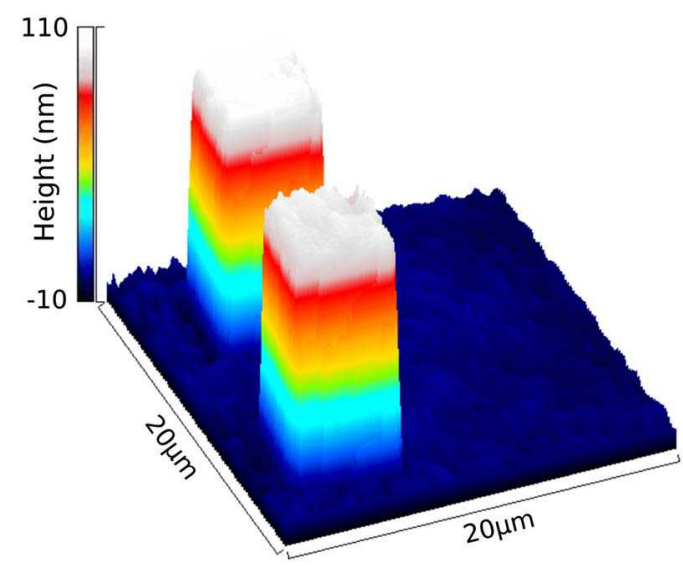

Fig. 8. VLSI test pattern of $100 \mathrm{~nm}$ height imaged with circular scanning $\left(\left|k_{i z}\right|=\right.$ constant, $\left.\theta=40^{\circ}\right)$.

in solving the $2 \pi$ phase ambiguity problem, as in classical spectral interferometry. The range of equivalent wavelengths reachable by this technique depends on the range of achievable $\theta$ angles. For a high NA, oil immersion microscope $(\mathrm{NA}=1.4$, $n=1.515$ ), $\cos \theta$ can vary from 1 to 0.382 , leading to an equivalent wavelength range of. $[\lambda ; 2.55 * \lambda]$. In our case with $\lambda_{0}=475 \mathrm{~nm}$, the equivalent wavelength can vary from 313 to $820 \mathrm{~nm}$. Otherwise, the measured height is modulated by $k_{i z}$, i.e., reduced by a $\cos \theta$ factor. The apparent height falls below $\Delta z_{\min }$ and the real height is found after correction by the $\cos \theta$ factor. Figure 8 illustrates this process when imaging the STR10-1000P silicon test pattern. The $100 \mathrm{~nm}$ high pitches cannot be directly imaged with immersion objectives, as the limitation of the height variation is given by: $\Delta z_{\min }=\lambda / 4=\lambda_{0} / 4 n_{w} \simeq 89 \mathrm{~nm}$ for water immersion and $78 \mathrm{~nm}$ for oil immersion. Using air immersion objective would increase this height limitation, albeit at the price of a lower lateral resolution, because of the lower NA. Figure 8 shows two pits correctly reconstructed.

Of course, if the sample is of unknown height, one cannot directly set the correct value for $\theta$ that permits unambiguous phase unwrapping. A solution is to use increasing values of the tilt angle, in order to get successive measurements of the same profile. However, this would lead to many measurements ( $\mathrm{N}$ circular scanning). A more general method is to perform an analogue to two-wavelength measurement in classical interferometry [33], but instead of controlling the optical frequency of the illumination, one tunes the spatial (angular spectrum) frequency. Figure $\underline{9}$ (blue-dashed curve) depicts the reconstructed profile with 40 holograms, and an illumination angle of $12^{\circ}$. The $k_{i z}$ modulation is too low, and the reconstructed profile height is not correct. The red curve on Fig. 9 shows the reconstructed profile taking into account these holograms, and also holograms recorded at an illumination angle of $40^{\circ}$, which permits a correct phase unwrapping. The profile is now reconstructed with the correct altitude. So, correct phase unwrapping is facilitated by

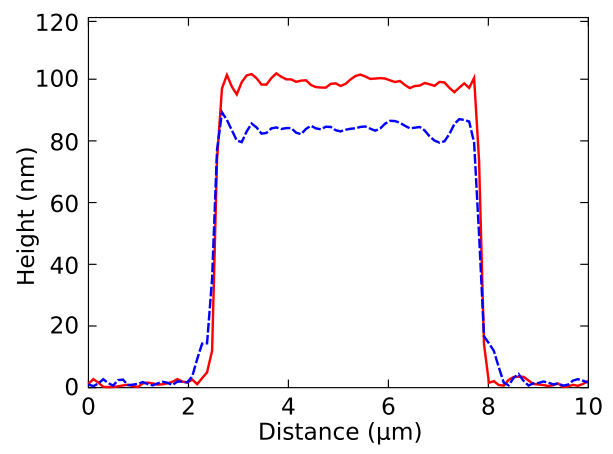

Fig. 9. Measurements of the VLSI target step height. Bluedashed curve: the unwrapping process failed and the height measurement is underestimated. Red curve: correct value estimated by double measurement.

control of the illumination angle. The technique is limited to shallow specimens; the sample must fit within the focal depth of observation. Studying deeper samples may be performed by increasing the observation wavelength or decreasing the objective NA, but this is detrimental to the resolution. Three-dimensional image stitching could constitute an alternate to observe deeper samples while keeping a high resolution.

\section{High-Resolution Imaging}

Tomographic diffractive microscopy permits to image samples with a high resolution, even when reconstructing samples under simplified assumptions related to the Born approximation [20,21,34]. With more elaborate approaches, the resolution can even be further improved [35]. These promising approaches are however ( $\overline{\mathrm{up}}$ to now) limited to very small samples because of numerical difficulties in the reconstruction algorithms [36]. We here illustrate the achievable resolution by our setup.

We consider the reflective surface of a Blu-ray disc, obtained by cleavage of the $0.1 \mathrm{~mm}$ protective polycarbonate layer. Such a sample presents interesting features, highlighting two advantages of tomographic diffractive microscopy, compared to more conventional approaches. First, the pits are very small: $130 \mathrm{~nm}$ width, with a minimum mark length of $149 \mathrm{~nm}$ for a single- or double-layer disc [37], below the resolution of conventional wide-field $\mathrm{mi}$ croscope $(\lambda / 2 \mathrm{NA}=170 \mathrm{~nm}$ in our case). Then, the distance between the pits is $190 \mathrm{~nm}$, close to this resolution, and the track pitch is $320 \mathrm{~nm}$ (larger than the resolution). The surface is almost flat, with a pit depth of about $60 \mathrm{~nm}$ (or $\lambda / 4 n$ with $n=1.6117$ the index of refraction of polycarbonate [38] at $\lambda=$ $405 \mathrm{~nm}$ the Blu-ray wavelength).

Because the pit width and depth and the pitch groove are small, it is difficult to focus on the object. A second advantage of tomographic diffractive microscopy is that the various holograms can be acquired without being exactly at focus. They are then backpropagated with a numerical sharpness metric, such as absolute gradient [39] or even a simple 


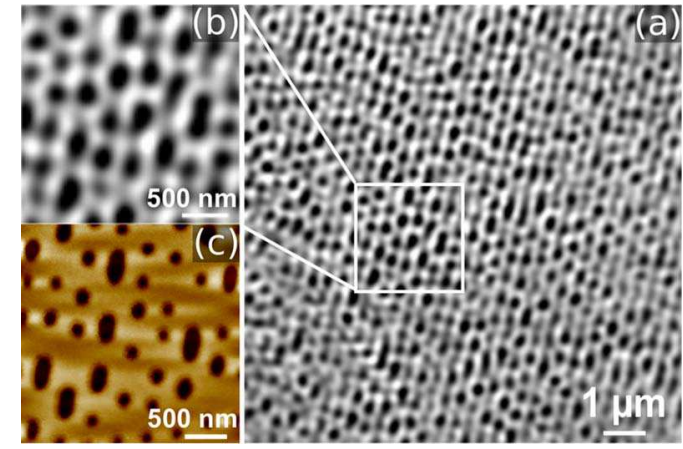

Fig. 10. (a) $2 \mathrm{D}$ amplitude imaging of a Blu-ray disc observed with tomographic diffractive microscopy, (b) enlarged view of (a), and (c) AFM topographic image of the same disc at same scale as (b).

criterium (max-min) on the integrated amplitude, depending on the sample [40]. After backpropagation at exact focus, the holograms can be merged and lead to the synthesized image.

Figure 10 shows the surface of an embossed Blu-ray disc imaged with our setup. Note that the pits are clearly identified, confirming the high lateral resolution achievable with this approach, despite the use of a simple Fourier reconstruction, which is not the best adapted for metallic surfaces. A 2.5 by $2.5 \mu \mathrm{m}$ inset is enlarged and compared at the same scale with an image of the same disc (albeit not the same zone), observed with AFM. The average depth of the pits is measured (on 10 pits) by AFM at $48 \pm 5 \mathrm{~nm}$. Such a small pit depth is not accurately measurable nor by our TDM (which has a excellent lateral resolution but which longitudinal resolution is lower than the phase imaging precision), nor by phase imaging because on such small samples, diffraction dominates, and the measured phase is not anymore directly linked to the profile. In order to get higher resolution images, reconstruction of the Blu-ray surface should be performed with nonlinear reconstruction methods, which can take into account the metallic nature of the surface and are better suited for smaller structures reconstruction [35,36]. An advantage of TDM with Fourier reconstruction, compared to the AFM we used or to these more advanced methods, is the much larger field of view.

\section{Conclusion and Perspectives}

We have developed a tomographic diffractive microscope in reflection, which is capable of an improved lateral resolution, while simultaneously delivering high-longitudinal precision for very shallow specimens (phase imaging), as well as improved $z$ resolution (tomographic imaging) for deeper ones. Associated with an automatic aberration compensation algorithm, the setup allows for reducing the spatial coherent noise and for facilitating the unwrapping process as well as the focusing process (numerical refocusing).

For sample features at the limit of resolution, phase imaging is affected by measurement errors due to diffraction effects. Furthermore, care should be taken if multiple reflections occur, as for example for deep metallic samples. The reconstruction methods used here are no longer valid and a more complex scheme should be used to include the effect of multiple scattering, such as iterative reconstruction procedures based on rigorous wave diffraction [41]. Polarization effects are also taken into account by such approaches, which helps distinguish structures below the classical limit of resolution.

For semitransparent samples, coupling tomographic imaging in transmission and in reflexion would permit to reach an isotropic resolution [42]. Similarly, the combination of phase imaging in transmission and in reflexion could help to discriminate small features at the limit of resolution. For birefringent samples, controlling the polarization of the illumination, as well as the angle of incidence, should allow for a precise measurement of the anisotropic properties of such samples [43].

Our setup may be useful to observe, for example, living cells growing on surfaces or biofilms. In such cases, photo-induced damages to the sample may be a concern, even if no fluorescence labeling is used. In this experiment, the power density on the sample has been estimated about $0.5 \mathrm{~mW} \cdot \mathrm{cm}^{-2}$, while a value of $0.1 \mathrm{~mW} \cdot \mathrm{cm}^{-2}$ has already been proven compatible with living cell imaging [16]. We therefore believe that, after optimization, our system may also be used for biological investigations.

Other possible applications of the method may be thin-film observations. While ellipsometry permits very precise measurement of the thickness and index of refraction of multilayered samples, precise measurement of local roughness or defects requires complementary observations. In general, research on surface coatings may benefit from this new technique, as for example, a study of the wear behavior of protective or hardening coatings [44]. Highresolution inspection of microelectronic devices or micromechanical systems may also benefit from this technique.

The authors gratefully acknowledge Laurent Vona (IS2M-Mulhouse) for the Blu-ray AFM images.

\section{References}

1. B. Kemper and G. von Bally, "Digital holographic microscopy for live cell applications and technical inspection," Appl. Opt. 47, A52-A61 (2008).

2. J. Goodman, Introduction to Fourier Optics (McGraw-Hill, 2008).

3. J. H. Massig, "Digital off-axis holography with a synthetic aperture," Opt. Lett. 27, 2179-2181 (2002).

4. C. Liu, Z. Liu, F. Bo, Y. Wang, and J. Zhu, "Super-resolution digital holographic imaging method," Appl. Phys. Lett. 81, 3143-3145 (2002).

5. M. Paturzo, F. Merola, S. Grilli, S. De Nicola, A. Finizio, and P. Ferraro, "Super-resolution in digital holography by a twodimensional dynamic phase grating," Opt. Express 16, 17107-17118 (2008)

6. V. Mico, Z. Zalevsky, P. García-Martínez, and J. García "Synthetic aperture superresolution with multiple off-axis holograms," J. Opt. Soc. Am. A 23, 3162-3170 (2006).

7. F. Charrière, A. Marian, F. Montfort, J. Kuehn, T. Colomb, E. Cuche, P. Marquet, and C. Depeursinge, "Cell refractive 
index tomography by digital holographic microscopy," Opt. Lett. 31, 178-180 (2006).

8. T. R. Hillman, T. Gutzler, S. A. Alexandrov, and D. D. Sampson, "High-resolution, wide-field object reconstruction with synthetic aperture Fourier holographic optical microscopy," Opt. Express 17, 7873-7892 (2009).

9. S. A. Alexandrov, T. R. Hillman, T. Gutzler, and D. D. Sampson, "Synthetic aperture Fourier holographic optical microscopy," Phys. Rev. Lett. 97, 168102 (2006).

10. X. Chen and S. Brueck, "Imaging interferometric lithography: approaching the resolution limits of optics," Opt. Lett. 24, 124-126 (1999).

11. J. R. Price, P. R. Bingham, and C. Thomas, Jr., "Improving resolution in microscopic holography by computationally fusing multiple, obliquely illuminated object waves in the Fourier domain," Appl. Opt. 46, 827-833 (2007).

12. J. Bühl, H. Babovsky, A. Kiessling, and R. Kowarschik, "Digital synthesis of multiple off-axis holograms with overlapping Fourier spectra," Opt. Commun. 283, 3631-3638 (2010).

13. M. Debailleul, B. Simon, V. Georges, O. Haeberlé, and V. Lauer, "Holographic microscopy and diffractive microtomography of transparent samples," Meas. Sci. Technol. 19, 074009 (2008).

14. B. Simon, M. Debailleul, V. Georges, V. Lauer, and O. Haeberlé, "Tomographic diffractive microscopy of transparent samples," Eur. Phys. J. Appl. Phys. 44, 29-35 (2008).

15. Y. Sung, W. Choi, C. Fang-Yen, K. Badizadegan, R. R. Dasari, and M. S. Feld, "Optical diffraction tomography for high resolution live cell imaging," in Novel Techniques in Microscopy (Optical Society of America, 2009).

16. Y. Cotte, F. Toy, P. Jourdain, N. Pavillon, D. Boss, P. Magistretti, P. Marquet, and C. Depeursinge, "Marker-free phase nanoscopy," Nat. Photonics 7, 113-117 (2013).

17. S. Vertu, J. Flügge, J.-J. Delaunay, and O. Haeberlé, "Improved and isotropic resolution in tomographic diffractive microscopy combining sample and illumination rotation," Central Eur. J. Phys. 9, 969-974 (2011).

18. W. Gorski and W. Osten, "Tomographic imaging of photonic crystal fibers," Opt. Lett. 32, 1977-1979 (2007).

19. D. Malacara, Optical Shop Testing (Wiley, 2007) Vol. 59.

20. M. Sarmis, B. Simon, M. Debailleul, B. Colicchio, V. Georges, J.-J. Delaunay, and O. Haeberlé, "High resolution reflection tomographic diffractive microscopy," J. Mod. Opt. 57, 740745 (2010).

21. B. Simon, M. Debailleul, A. Beghin, Y. Tourneur, and O. Haeberlé, "High resolution tomographic diffractive microscopy of biological samples," J. Biophotonics 3, 462-467 (2010).

22. J. Kühn, F. Charrière, T. Colomb, E. Cuche, F. Montfort, Y. Emery, P. Marquet, and C. Depeursinge, "Axial subnanometer accuracy in digital holographic microscopy," Meas. Sci. Technol. 19, 074007 (2008).

23. A. Stadelmaier and J. H. Massig, "Compensation of lens aberrations in digital holography," Opt. Lett. 25, 1630-1632 (2000).

24. W. Qu, C. Choo, V. Singh, Y. Yingjie, and A. Asundi, "Quasiphysical phase compensation in digital holographic microscopy," J. Opt. Soc. Am. 26, 2005-2011 (2009).

25. P. Ferraro, S. De Nicola, A. Finizio, G. Coppola, S. Grilli, C. Magro, and G. Pierattini, "Compensation of the inherent wave front curvature in digital holographic coherent microscopy for quantitative phase-contrast imaging," Appl. Opt. 42, 1938-1946 (2003).

26. T. Colomb, E. Cuche, F. Charrière, J. Kühn, N. Aspert, F. Montfort, P. Marquet, and C. Depeursinge, "Automatic procedure for aberration compensation in digital holographic microscopy and applications to specimen shape compensation," Appl. Opt. 45, 851-863 (2006).

27. F. Charrière, T. Colomb, F. Montfort, E. Cuche, P. Marquet, and C. Depeursinge, "Shot-noise influence on the reconstructed phase image signal-to-noise ratio in digital holographic microscopy,” Appl. Opt. 45, 7667-7673 (2006).

28. X. Kang, "An effective method for reducing speckle noise in digital holography," Chin. Opt. Lett. 6, 100-103 (2008).

29. G. Parry and J. Dainty, "Laser speckle and related phenomena," in Topics in Applied Physics Series (Springer, 1984), Vol. 9.

30. L. Rong, W. Xiao, F. Pan, S. Liu, and R. Li, "Speckle noise reduction in digital holography by use of multiple polarization holograms," Chin. Opt. Lett. 8, 653-655 (2010).

31. P. Bon, G. Maucort, B. Wattellier, and S. Monneret, "Quadriwave lateral shearing interferometry for quantitative phase microscopy of living cells," Opt. Express 17, 13080-13094 (2009).

32. Z. Duan, Y. Miyamoto, and M. Takeda, "Dispersion-free absolute interferometry based on angular spectrum scanning," Opt. Express 14, 655-663 (2006).

33. K. Creath, "Step height measurement using two-wavelength phase-shifting interferometry," Appl. Opt. 26, 2810-2816 (1987).

34. M. Debailleul, V. Georges, B. Simon, R. Morin, and O. Haeberlé, "High-resolution three-dimensional tomographic diffractive microscopy of transparent inorganic and biological samples," Opt. Lett. 34, 79-81 (2009).

35. G. Maire, F. Drsek, J. Girard, H. Giovannini, A. Talneau, D. Konan, K. Belkebir, P. C. Chaumet, and A. Sentenac, "Experimental demonstration of quantitative imaging beyond Abbe's limit with optical diffraction tomography," Phys. Rev. Lett. 102, 213905 (2009).

36. G. Maire, Y. Ruan, T. Zhang, P. C. Chaumet, H. Giovannini, D. Sentenac, A. Talneau, K. Belkebir, and A. Sentenac, "Highresolution tomographic diffractive microscopy in reflection configuration," J. Opt. Soc. Am. A 30, 2133-2139 (2013).

37. "White paper blu-ray disctm format 3rd edition, 2012," http:// blu-raydisc.com/.

38. "Refractive index database 2013" http://refractiveindex.info.

39. P. Langehanenberg, B. Kemper, D. Dirksen, and G. Von Bally, "Autofocusing in digital holographic phase contrast microscopy on pure phase objects for live cell imaging," Appl. Opt. 47, D176-D182 (2008).

40. F. Dubois, C. Schockaert, N. Callens, and C. Yourassowsky, "Focus plane detection criteria in digital holography microscopy by amplitude analysis," Opt. Express 14, 5895-5908 (2006).

41. S. Arhab, G. Soriano, K. Belkebir, A. Sentenac, and H. Giovannini, "Full wave optical profilometry," J. Opt. Soc. Am. A 28, 576-580 (2011).

42. V. Lauer, "New approach to optical diffraction tomography yielding a vector equation of diffraction tomography and a novel tomographic microscope," J. Microsc. 205, 165-176 (2002).

43. Y. Kim, J. Jeong, J. Jang, M. W. Kim, and Y. Park, "Polarization holographic microscopy for extracting spatio-temporally resolved Jones matrix," Opt. Express 20, 9948-9955 (2012).

44. H. Liu, J. Bailleul, B. Simon, M. Debailleul, P. Henry, M.-J. Pac, and O. Haeberé, "Tomographic diffractive microscopy and multiview profilometry," in Focus on Microcopy (Maastricht UMC+, 2013), p. 155, http://www.focusonmicroscopy .org/2013/index.html. 